\title{
Research Article \\ Effect of Physical Activity on Blood Pressure Distribution among School Children
}

\author{
Anisa M. Durrani and Waseem Fatima \\ Department of Home Science, Aligarh Muslim University, India \\ Correspondence should be addressed to Anisa M. Durrani; anisamd@gmail.com
}

Received 9 May 2014; Revised 11 December 2014; Accepted 9 February 2015

Academic Editor: Jennifer L. Freeman

Copyright (C) 2015 A. M. Durrani and W. Fatima. This is an open access article distributed under the Creative Commons Attribution License, which permits unrestricted use, distribution, and reproduction in any medium, provided the original work is properly cited.

\begin{abstract}
The present study analyzed the relationship between physical activity and blood pressure in 701 school children aged 12-16 years (girls $=338$, boys $=363$ ). During the baseline examination, systolic blood pressure $(\mathrm{SBP})$ and diastolic blood pressure $(\mathrm{DBP})$, height, weight, and 24-hour recall of the working day activity with duration were recorded. Total activity score and type of activity were calculated by weighing the activity level. Mean, standard deviation, and correlation coefficient were calculated by using SPSS 12.0 version. The results revealed that rise in blood pressure was directly proportional to the increase in age. The range of systolic blood pressure was found to be high in low risk blood pressure (LBP) group than in elevated blood pressure (EBP) group showing direct association of activity level and systolic blood pressure. Physical activity score was found to be more in LBP group than in EBP group. Our results support the hypothesis that SBP is independently related to the level of habitual physical activity in children.
\end{abstract}

\section{Introduction}

There is a strong, continuous, and independent relationship between blood pressure and cardiovascular disease and allcause mortality. Physical inactivity is strongly positively associated with hypertension, and intervention studies have demonstrated that increased physical activity is effective in the treatment of high blood pressure in a variety of populations [1]. Several studies have provided ample evidence that hypertension in adults has its onset in childhood which has caused growing concern with monitoring arterial BP in children in the last few decades [2]. Therefore, the trend in blood pressure in children with respect to increase in age may be important predictors of subsequent trend in adult hypertension. A number of physical activities and intervention studied performed in children and adolescents described the influence of physical activity pattern and blood pressure $[3,4]$. Several studies have reported useful data on the benefits of exercise, health, and nutritional program in children $[5,6]$. However, to the best of our knowledge, relationships between physical activity and hypertension have not been studied in Indian context.
Physical activity and health behavior are tracked into adulthood; thus, it is important to study blood pressure distribution among children in school and community setting for designing and implementing effective policy and program to promote physical activity in the young people. In the present study, the relationship of blood pressure and physical activity of school children has been studied.

\section{Material and Methods}

This cross-sectional study was conducted in city of Aligarh among school children aged $12-16$ years. The total population of Aligarh in this age group was found to be 46.850 (Municipal Corporation of Aligarh, 2004-2005).

Considering $11.7 \%$ prevalence of hypertension among adolescence [7] and with $20 \%$ possible error, the sample size was calculated to be 755 by using the formula $N=$ $4 \mathrm{pq} / \mathrm{L}^{2}$. A sample of 701 school children ranging in age 12-16 years participated in the study, with response rate of $92.84 \%$. Four schools were selected based on the consent and active cooperation of the school authorities, by stratified 
random sampling by dividing the city into four specific zones on the geographical layout to ensure that the study was representative of whole city. Consent was obtained from parents of all the selected samples of school children.

The age was determined to the nearest birth date from the school registration record. Blood pressure measurements (BP) were taken with a mercury sphygmomanometer and stethoscope. The cuff used was adapted to the arm circumferences and was inflated to a level at which the distal arterial pulse was not palpable. Three consecutive readings of blood pressure after 5 minutes of rest at two minutes of interval were taken for both systolic blood pressure (SBP) and diastolic blood pressure (DBP) and their mean values were used in subsequent analysis. The measurements were taken by the researcher during the school hours, mostly in the morning in sitting position with the arm at the level of the heart, in a quiet isolated setting.

On the basis of these readings, 5th and 95th percentile were computed for each age group, genderwise for both systolic and diastolic blood pressure. Blood pressure ranging from 60 to $75 \mathrm{mmHg}$ (DBP) and 76 to $100 \mathrm{mmHg}$ (SBP), equal to or below 25th percentile, is considered for the low risk pressure group (LBP), and pressure range from 85 to $100 \mathrm{mmHg}$ (DBP) and 120 to $140 \mathrm{mmHg}$ (SBP), equal to or greater than 95th percentile, is considered for the high risk or elevated pressure group (EBP).

In the LBP group $81(11.6 \%)$ adolescents were identified and in EBP group 66 (9.4\%) adolescents were identified. From these adolescent populations an equal number of boys and girls from LBP and EBP group were selected from each age group. While comparing the EBP and LBP groups according to age and sex, the least number of five students was observed at the age of 12 years in girls. In order to reduce the influence of confounding variables in the two groups, the children were also matched with age, sex, and number for the purpose of comparing and evaluating the results between two pressure groups. A total number of 100 students were selected through purposive sampling method, comprising 50 boys and 50 girls. As only five girls were identified in the low mean pressure group (LBP) in 12-year age group, equal number of 5 boys and 5 girls from 12 to 16 years of age comprising 50 adolescents ( 25 boys and 25 girls) were selected in LBP group. The equal numbers of 50 adolescents ( 25 boys and 25 girls) constituted the elevated blood pressure (EBP) group, thereby matching the two pressure groups (LBP and EBP) with age, sex, and number. In the final sample of 100 adolescents, an in-depth study was carried out. The comprehensive data was collected through a validated questionnaire to find out physical activity pattern.

The 24-hour recall of the previous working day activities and their duration was recorded. Total activity score and the type of activity for the two groups (LBP and EBP) were also calculated by weighing the activity level in the following manner. Time spent in sleeping and in the very light activity categories was multiplied by a factor one, light activity by a factor two, moderate activity by three, and strenuous activity by a factor four. Physical activity score for total activity was calculated by summation of these scores. The collected data were stored in a database (Microsoft Excel 2003) and mean, standard deviation (SD), and correlation coefficient were calculated by using SPSS 12 to establish significance between the LBP and EBP adolescent. The results were seen at 5\% level of significance and $P$ value $<0.05$ was considered to be statistically significant.

\section{Results}

A total of 701 school children between 12 and 16 years constituting 363 (51.78\%) boys and 338 (48.21\%) girls were included for the study. Table 1 presents the range of blood pressure $(\mathrm{mmHg})$ in LBP and EBP groups. The range was more or less the same for diastolic blood pressure in both the LBP and EBP groups. In case of systolic blood pressure, the range was found to be more in LBP group than in EBP group without any significance. The percentile value of both systolic blood pressure (Figure 1) and diastolic blood pressure (Figure 2) revealed that the rise in blood pressure was directly proportional to the increase in the age and was found to be highly significant $(P<0.05)$.

The mean and standard deviation of one-day physical activity (hrs/day) and energy expenditure in LBP and EBP group are presented in Table 2. The total activity score of LBP and EBP group was $37.67 \pm 4.4$ and $35.41 \pm 6.05$, respectively, $P<0.05$ level. Children of LBP did more moderate activity than EBP children and highly significant difference at $P<$ 0.001 level was observed in the two pressure groups. However, no significant difference was found in time spent in sleeping, very light activity, and light and strenuous activity.

The physical activity correlate of blood pressure seen in Table 3 indicates a positive association of moderate activity and energy expenditure with diastolic blood pressure of boys in EBP group at $P<0.05$ level. In LBP group, no association between physical activity and blood pressure was found in boys.

In EBP group, no association was found between physical activity and blood pressure among girls. But LBP group showed direct association of strenuous activity with diastolic blood pressure and it was found to be significant at $(P<0.05)$ level.

\section{Discussion}

The pathological processes associated with development of cardiovascular disease begin early in life. For example, elevated blood pressure (BP) can be seen in childhood and is tracked into adulthood. The relationship between physical activity (PA) and BP in adults is well established, but findings in children have been inconsistent with few studies.

Thakor et al., 2004, found that both SBP and DBP were significantly associated with outdoor playing taking the whole sample as one, but not in different sex or age group, except that the SBP was significantly correlated with outdoor playing in 10-13 years of age group in boys [8]. Hansen and Hyidebebrendt 1989 found an inverse correlation between blood pressure and physical fitness [9]. Klesges et al., 1990, found no consistent relationship between various childhood activity factors and cardiovascular risk factors, weight and blood pressure [10]. Jenner et al., 1992, reported that the 
TABLE 1: Range of blood pressure $(\mathrm{mmHg})$ in LBP and EBP groups.

\begin{tabular}{lcc}
\hline Group & Diastolic blood pressure & Systolic blood pressure \\
\hline LBP & $60-75$ & $76-100$ \\
EBP & $85-100$ & $120-140$ \\
\hline
\end{tabular}

TABLE 2: Mean and standard deviation of one-day physical activity (hrs/day) and energy expenditure in both boys and girls of LBP and EBP groups.

\begin{tabular}{lccc}
\hline Physical activity $(\mathrm{hrs})$ & LBP $(n=50)$ & EBP $(n=50)$ & $Z$ \\
\hline sleep & $8.21(1.22)$ & $8.28(1.15)$ & 0.60 \\
Very light & $1.58(1.1)$ & $1.59(1.27)$ & 0.50 \\
Light & $22.46(5.3)$ & $21.72(4.9)$ & 0.080 \\
Moderate & $4.2(3.3)$ & $2.37(2.57)$ & $1.9^{* *}$ \\
Strenuous & $1.15(2.3)$ & $1.63(2.56)$ & 0.60 \\
\hline Total activity score & $37.67(4.4)$ & $35.41(6.05)$ & $1.4^{*}$ \\
\hline
\end{tabular}

Figures in parenthesis indicate standard deviation.

${ }^{*} P<0.05$.

${ }^{* *} P<0.001$.

number of physical active days per week was negatively correlated with DBP in girls, while relationship with SBP in girls and SBP and DBP in boys was nonsignificant [11].

Leary et al., 2008, concluded that higher levels of physical activity were associated with lower blood pressure, and results suggested that the volume of activity may be more important than the intensity [6]. In the present study also girls in LBP group showed significant association with physical activity and blood pressure.

The physical activity correlate of blood pressure indicates a positive association of moderate activity and energy expenditure with diastolic blood pressure of boys in EBP group at $P<0.05$ level. But in comparison to girls, the boys in LBP group showed no association between physical activity and blood pressure.

A number of studies have emerged recently describing the influence of lifestyle interventions on various risk factors associated with high blood pressure in overweight and obese children and adolescence. The benefits of physical activity in the prevention and treatment of high blood pressure in adults have been very well described by Hagberg et al. and Whelton et al. $[3,12]$.

Regular physical activity and restriction of sedentary activity will improve efforts at weight management and may prevent an excess increase in blood pressure over time. A recent meta-analysis study of Kelley et al., which combined 12 randomized trials, for a total of 1266 children and adolescents, concluded that physical activity leads to a small but not statistically significant decrease in blood pressure. However, both regular physical activity and decreasing sedentary activities (such as watching television and playing video or electronic games) are important components of pediatric obesity treatment and prevention [13].

Public health approaches, such as reducing calories, saturated fat, and salt in processed foods and increasing

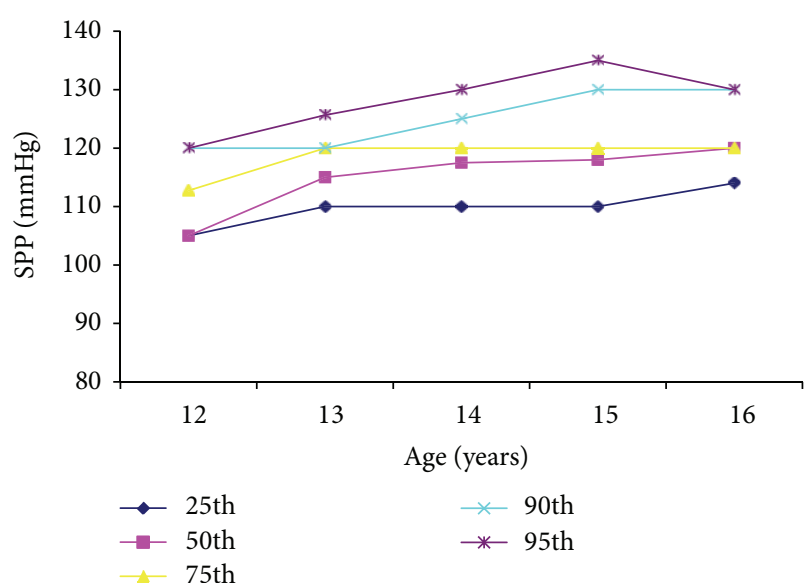

FIGURE 1: Percentile distribution of systolic blood pressure.

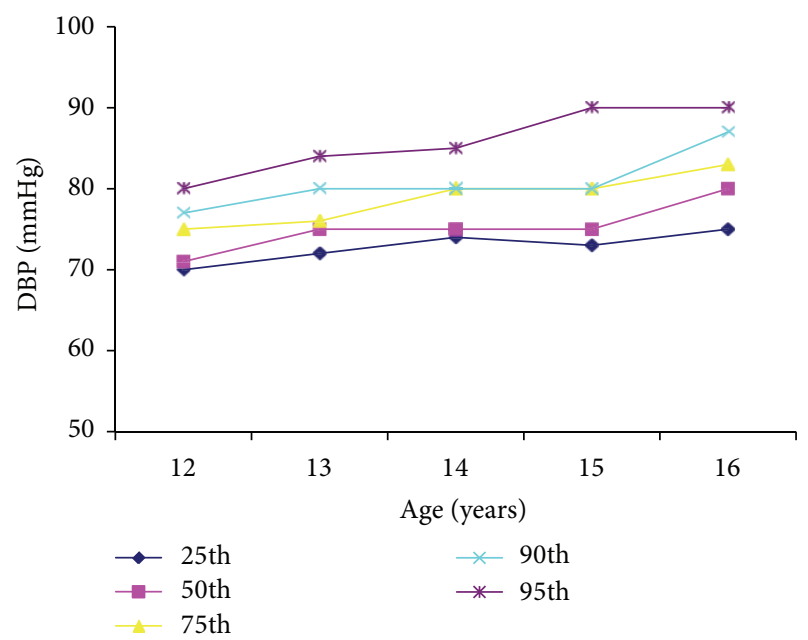

FIGURE 2: Percentile distribution of diastolic blood pressure.

community/school opportunities for physical activity, can achieve a downward shift in the distribution of a population's $\mathrm{BP}$; thus potentially reducing morbidity, mortality, and the lifetime risk of an individual's becomes hypertensive.

When public health intervention strategies address the diversity of racial, ethnic, cultural, linguistic, religious, and social factors in the delivery of their services, the likelihood of their acceptance by the community increases. These public health approaches can provide an attractive opportunity to interrupt and prevent the continuing costly cycle of managing hypertension and its complications.

In conclusion, higher levels of PA were associated with lower BP, and results suggested that the volume of activity may be more important than the intensity.

\section{Conflict of Interests}

The authors declare that there is no conflict of interests regarding the publication of this paper. 
TABLE 3: Correlation coefficient of blood pressure of LBP and EBP group in boys and girls by physical activity and energy expenditure.

\begin{tabular}{|c|c|c|c|c|c|c|c|c|}
\hline \multirow{3}{*}{ Physical activity } & \multicolumn{4}{|c|}{$\operatorname{LBP}(n=50)$} & \multicolumn{4}{|c|}{$\operatorname{EBP}(n=50)$} \\
\hline & \multicolumn{2}{|c|}{ DBP } & \multicolumn{2}{|c|}{ SBP } & \multicolumn{2}{|c|}{ DBP } & \multicolumn{2}{|c|}{ SBP } \\
\hline & Girls (25) & Boys (25) & Girls (25) & Boys (25) & Girls (25) & Boys (25) & Girls (25) & Boys (25) \\
\hline Sleep & 0.00 & -0.17 & 0.21 & -0.01 & -0.01 & -0.11 & -0.30 & -0.36 \\
\hline Very light & 0.10 & -0.07 & -0.07 & -0.39 & -0.20 & -0.14 & -0.10 & 0.25 \\
\hline Light & -0.10 & -0.02 & -0.02 & -0.22 & -0.18 & 0.21 & 0.17 & 0.34 \\
\hline Moderate & -0.17 & -0.16 & 0.04 & -0.01 & -0.01 & $0.42^{*}$ & -0.08 & 0.27 \\
\hline Strenuous & $0.48^{*}$ & 0.19 & 0.13 & 0.24 & 0.17 & -0.09 & -0.06 & -0.30 \\
\hline Total activity score & -0.10 & -0.05 & 0.09 & 0.30 & -0.14 & 0.23 & 0.09 & 0.16 \\
\hline
\end{tabular}

Figures in parenthesis indicate number of boys and girls.

${ }^{*} P<0.05$.

\section{References}

[1] J. Stamler, R. Stamler, and J. D. Neaton, "Blood pressure, systolic and diastolic, and cardiovascular risks: US population data," Archives of Internal Medicine, vol. 153, no. 5, pp. 598-615, 1993.

[2] N. J. Wareham, M.-Y. Wong, S. Hennings et al., "Quantifying the association between habitual energy expenditure and blood pressure," International Journal of Epidemiology, vol. 29, no. 4, pp. 655-660, 2000.

[3] S. P. Whelton, A. Chin, X. Xin, and J. He, "Effect of aerobic exercise on blood pressure: a meta-analysis of randomized, controlled trials," Annals of Internal Medicine, vol. 136, no. 7, pp. 493-503, 2002.

[4] G. A. Kelley and K. S. Kelley, "Aerobic exercise and resting blood pressure in women: a meta-analytic review of controlled clinical trials," Journal of Women's Health and Gender-Based Medicine, vol. 8, no. 6, pp. 787-803, 1999.

[5] G. A. Kelley, K. A. Kelley, and Z. Vu Tran, "Aerobic exercise and resting blood pressure: a meta-analytic review of randomized, controlled trials," Preventive Cardiology, vol. 4, no. 2, pp. 73-80, 2001.

[6] S. D. Leary, A. R. Ness, G. D. Smith et al., "Physical activity and blood pressure in childhood: findings from a population-based study," Hypertension, vol. 51, no. 1, pp. 92-98, 2008.

[7] S. L. Chadha, R. Tandon, S. Shekhawat, and N. Gopinath, "An epidemiological study of blood pressure in school children (514 years) in Delhi," Indian Heart Journal, vol. 51, no. 2, pp. 178$182,1999$.

[8] H. G. Thakor, P. Kumar, and V. K. Desai, "Effect of physical activity and mental activity on blood pressure," The Indian Journal of Pediatrics, vol. 71, no. 4, pp. 307-312, 2004.

[9] S. H. Hansen and M. Hyidebebrendt, "Familiar aggregation of BP in children," Scandinavian Journal of Clinical and Laboratory Investigation, vol. 49, pp. 55-57, 1989.

[10] R. C. Klesges, C. K. Haddock, and L. H. Eck, "A multimethod approach to the measurement of childhood physical activity and its relationship to blood pressure and body weight," Journal of Pediatrics, vol. 116, no. 6, pp. 888-893, 1990.

[11] D. A. Jenner, R. Vandongen, and L. J. Beilin, "Relationships between blood pressure and measures of dietary energy intake, physical fitness, and physical activity in Australian children aged 11-12 years," Journal of Epidemiology and Community Health, vol. 46, no. 2, pp. 108-113, 1992.

[12] J. M. Hagberg, J.-J. Park, and M. D. Brown, "The role of exercise training in the treatment of hypertension: an update," Sports Medicine, vol. 30, no. 3, pp. 193-206, 2000.
[13] G. A. Kelley, K. S. Kelley, and Z. V. Tran, "The effects of exercise on resting blood pressure in children and adolescents: a metaanalysis of randomized controlled trials," Preventive Cardiology, vol. 6, no. 1, pp. 8-16, 2003. 


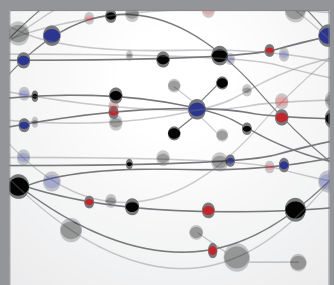

The Scientific World Journal
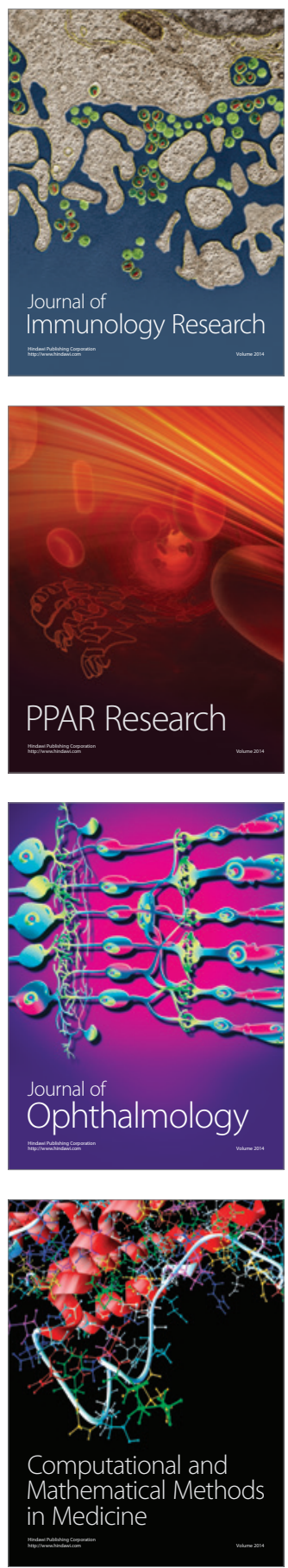

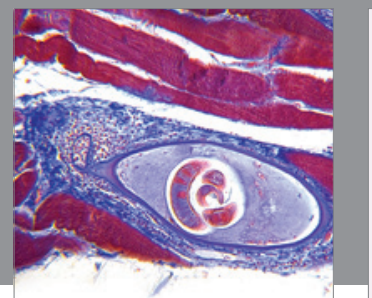

Gastroenterology

Research and Practice
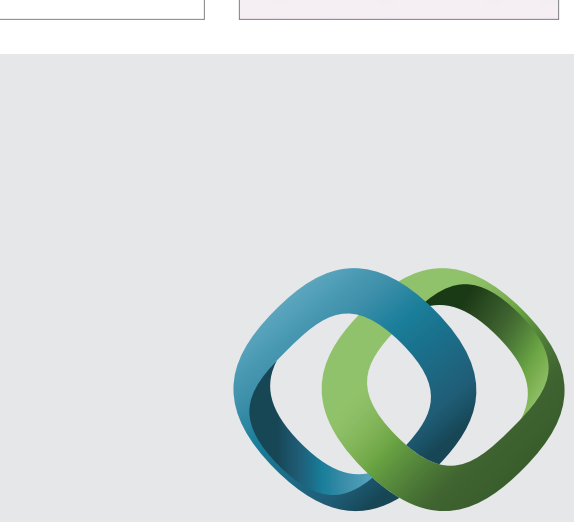

\section{Hindawi}

Submit your manuscripts at

http://www.hindawi.com
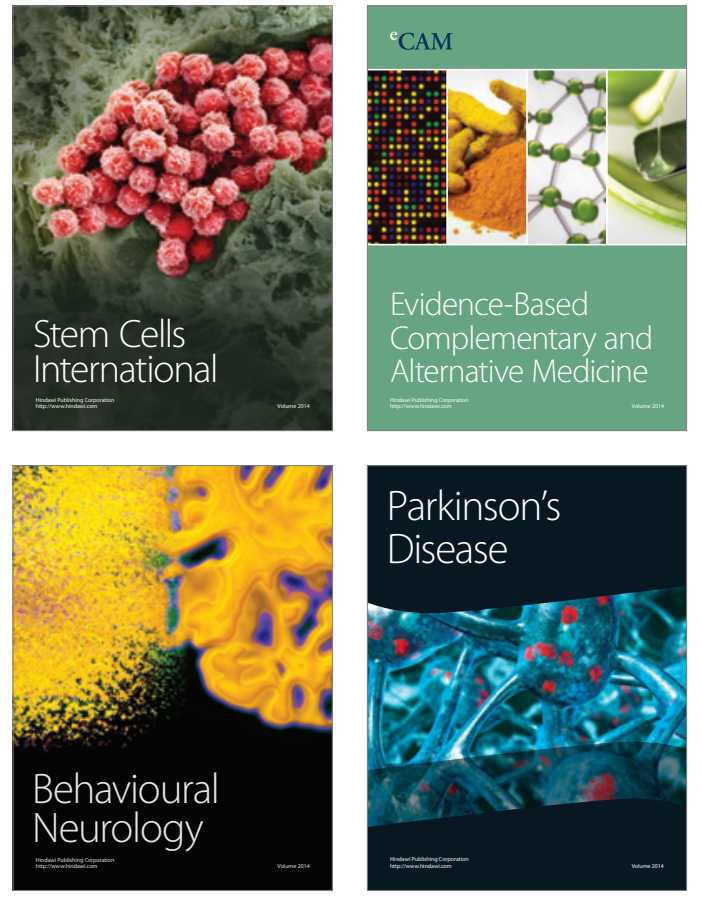
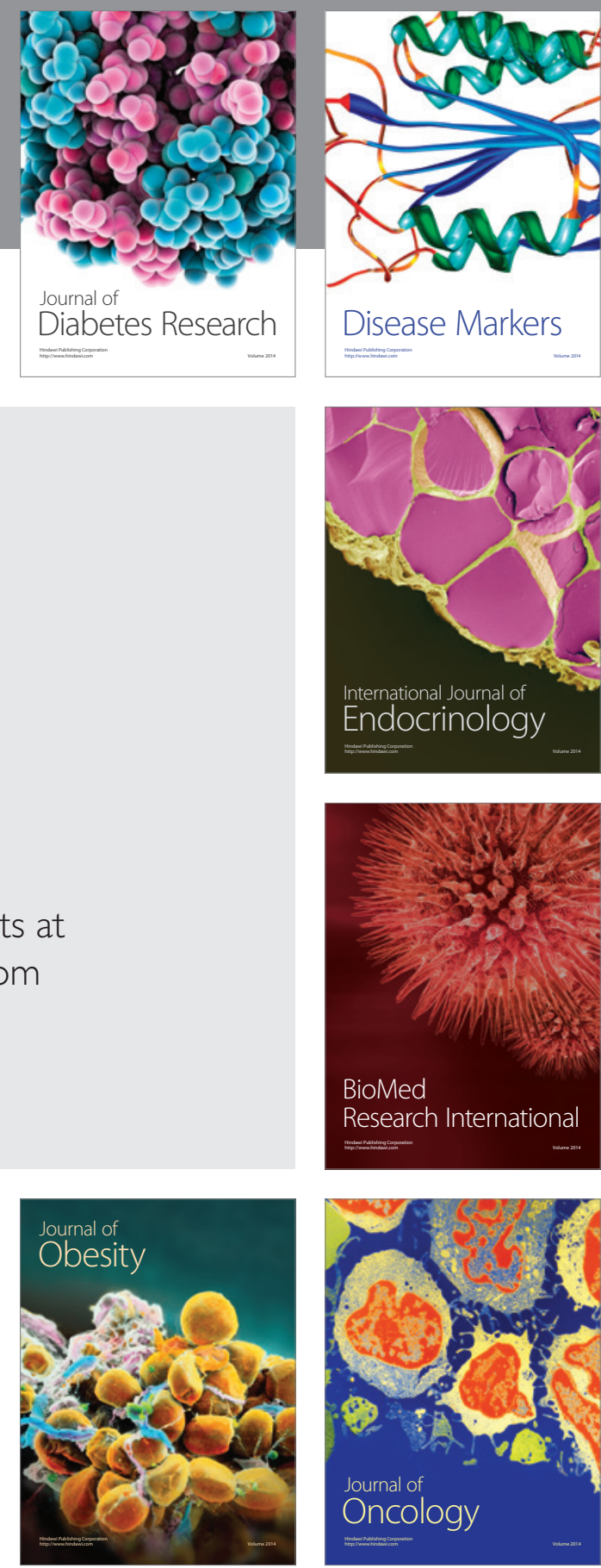

Disease Markers
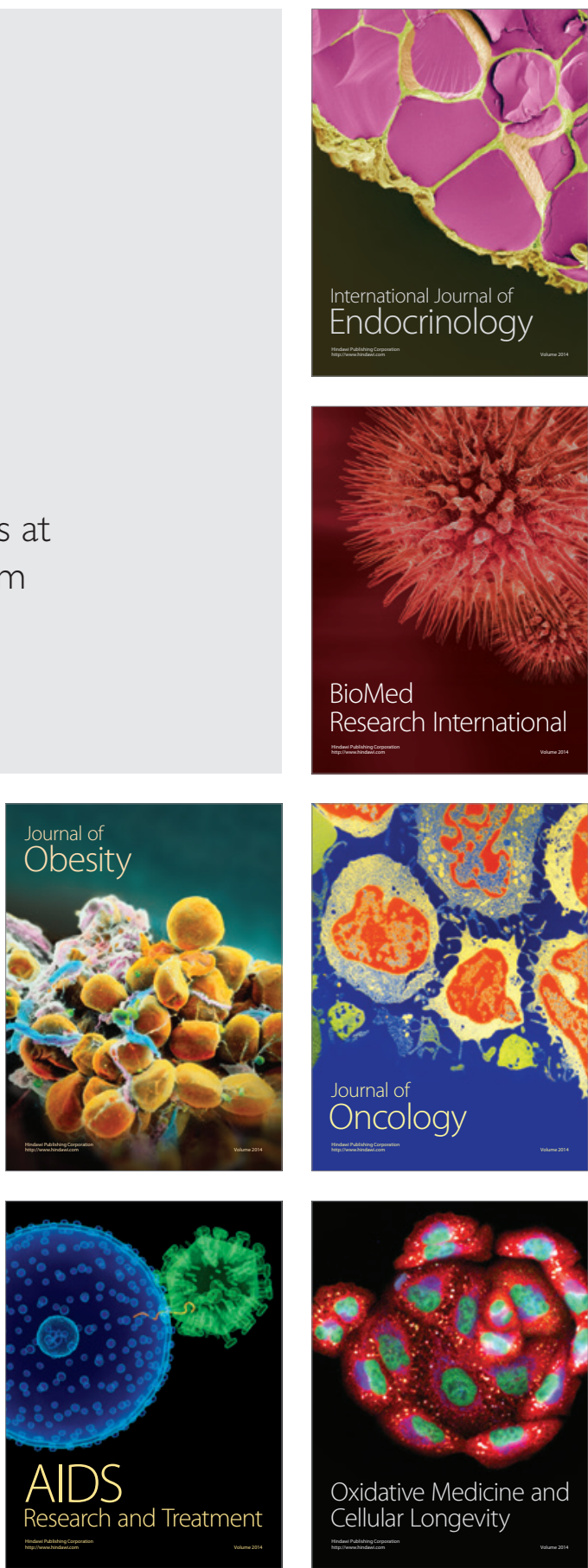